P. J. Moody, G. M. Reed, A. W. Roscoe and P. J. Collins, A lattice of conditions on topological spaces II . . . . . . . . . . . . . . . . . . . . . . .
G. KezK. Mazur, $F_{\sigma}$-ideals and $\omega_{1} \omega_{1}^{*}$-gaps in the Boolean algebras $P(\omega) / I$. . . . . . . . . 103-111

D. Simson, A splitting theorem for multipeak path algebras . . . . . . . . . 113-137

J. Nikiel and E. D. Tymchatyn, Sets of end-points and ramification points in

dendroids . E. D. . . 139-146

M. Gar a y, Strong cellularity and global asymptotic stability . . . . . . . . . 147-154

The FUNDAMENTA MATHEMATICAE publishes papers devoted to Set Theory, Topology, Mathematical Logic and Foundations, Real Functions, Measure and

Integration, Abstract Algebra

Each volume consists of three separate issues

Manuscripts and editorial correspondence should be addressed to: FUNDAMENTA MATHEMATICAE

Śniadeckich 8, P.O. Box 137, 00-950 Warszawa, Poland, telex 816112 PANIM PL

Papers for publication should be submitted in two typewritten (double spaced) copies and contain a short abstract. A complete list of all handwritten symbols with indications for the printer should be enclosed. Special typefaces should be indicated according to the following code: script letters by encircling the typed Roman letter in black, German letters-by typing the Roman equivalent and underlining in green, boldface letters - by straight black underlining. The authors will receive 50 reprints of their articles.

Correspondence concerning subscriptions, library exchange and back numbers should be sent to:

INSTITUTE OF MATHEMATICS, POLISH ACADEMY OF SCIENCES Śniadeckich 8, P.O. Box 137, 00-950 Warszawa, Poland, telex 816112 PANIM PL

(c) Copyright by Instytut Matematyczny PAN, Warszawa 1991

Published by PWN - Polish Scientific Publishers

ISBN $83-01-10423-6 \quad$ ISSN $0016-2736$

\section{A lattice of conditions on topological spaces II}

\section{by}

\section{P. J. Moody, G. M. Reed, A. W. Roscoe and P. J. Collins (Oxford)}

Abstract. Earlier work of some of the authors is developed to give new criteria for Abstract. Earler wacompactness. The point countable base conjecture is shown to be true for developability and paracompactness. The point countable base conjecture is show seces and spaces with density $\leqslant \omega_{1}$. certain classes of spaces, in particular for semi-stratifiable spaces and spaces with density $\leqslant \omega_{1}$.
The structure of any counterexample is also investigated. The notion of an acyclic monotonically normal operator on a space $X$ is introduced and is shown to be equivalent to the condition "chain (F)" which is shown to imply that $X$ is a $\mathrm{K}_{0}$-space.

0. Introduction. The aim of this paper is to develop earlier work of some of the authors and M. E. Rudin [6], [7]. We shall be investigating variants on $(F)$ and $(G)$ of the earlier papers which we now specify. They apply to topological spaces $X$ for each element $x$ of which a family $W(x)$ of subsets containing $x$ is given. Let $\mathscr{W}=\{W(x)$ : $x \in X\}$. We say that $\mathscr{W}$ satisfies $(\mathrm{F})$ when it satisfies

(F) if $x \in U$ and $U$ is open, then there exists an open $V=V(x, U)$ containing $x$ such that $x \in W \subseteq U$ for some $W \in W(y)$ whenever $y \in V$.

Any topological space clearly has such a family of open sets satisfying $(F)$. All the conditions we discuss are determined by placing restrictions on $\mathscr{W}$.

We say that $\mathscr{W}$ satisfies $(\mathrm{G})$ if $\mathscr{W}$ satisfies $(\mathrm{F})$ and $W(x)$ is countable for every $x \in X$. $\mathscr{W}$ is said to satisfy open $(\mathrm{F})$, or chain $(\mathrm{F})$, if $\mathscr{W}$ satisfies $(\mathrm{F})$ and each element of each $W(x)$ is open, or each $W(x)$ is a chain with respect to inclusion. We similarly modify $(\mathrm{G})$ and, in particular, say that $\mathscr{W}$ satisfies decreasing $(\mathrm{G})$ if $\mathscr{W}$ satisfies $(\mathrm{G})$ and for each $x$, there is an enumeration of $W(x)$ as $\{W(n, x): n \in N\}$ such that $W(n+1, x) \subseteq W(n, x)$ for each $n$ (where $N$ is the set of natural numbers). Furthermore, we say $\mathscr{W}$ satisfies uniform open $(\mathrm{G})$ if $\mathscr{W}$ satisfies open $(\mathrm{G})$ and for each $x \in X$, every infinite subset of $W(x)$ forms a local base at $x\left({ }^{1}\right)$.

1980 Mathematics Subject Classification: Primary 54D15, 54D18, 54E30, 54E65; Secondary $54 \mathrm{C} 99,54 \mathrm{E} 20$.

Key words and phrases: developable, paracompact, point countable base, monotonically normal.

(1) A local base at $x$ is a collection $O B$ of open neighbourhoods of $x$ such that for each neighbourhood $N$ of $x$ there exists a $B \in \mathscr{B}$ with $B \subseteq N$. 
The paper is split into three essentially disjoint sections the first of which establishes that if a space has $\mathscr{W}$ satisfying uniform open $(G)$ then it is developable (Theorem 1 ). In the second section the point countable base conjecture is investigated. The conjecture, which was first posed as a question in [6], is that every space with $\mathscr{W}$ satisfying open (G) has a point countable base. Pointed open covers are introduced and it is shown (Lemma 2) that the conjecture is true if and only if every space with $\mathscr{W}$ satisfying open (G) has a dense, point countable, pointed open cover. This result is used to establish that for certain classes of spaces the conjecture is true (Theorem 7). The structure of any counterexample is investigated and in particular it is shown that any counterexample contains a non-empty subspace, every non-empty open subset of which is a counterexample (Theorem 9).

In the final section we introduce the notion of an acyclic monotonically normal operator and establish that a space has such an operator if and only if the space has $\mathscr{W}$ satisfying chain (F) (Theorem 11). From this it is deduced that generalised order (GO) spaces and elastic spaces have $\mathscr{W}$ satisfying chain (F) (Theorem 12). We also show that a space with $\mathscr{W}$ satisfying chain $(\mathrm{F})$ is a $\mathrm{K}_{0}$-space (Theorem 13) and state the "unified paracompactness theorem" indicated in [6] (Theorem 14).

All our spaces will satisfy the $\mathrm{T}_{1}$ separation axiom. $A^{\circ}$ will denote the interior and $\bar{A}$ the closure of the set $A$. Finally, as mentioned above, $N$ will denote the set of natural numbers.

1. When $\mathscr{W}$ satisfies uniform open $(G)$. We first note that if $\mathscr{W}$ satisfies open decreasing $(\mathrm{G})$ then $\mathscr{W}$ satisfies uniform open $(\mathrm{G})$. In $[6]$ it was established that a space having $\mathscr{W}$ satisfying open decreasing $(G)$ is metrisable. The proof of this result consisted in showing such spaces were collectionwise normal and developable, and then applying Theorem 10 of R. H. Bing [2]. Here we prove the following result.

THEOREM. $A$ space is metacompact and developable if and only if it has $\mathscr{W}$ satisfying uniform open $(\mathrm{G})$.

The concept of $\mathscr{W}$. satisfying uniform open $(\mathrm{G})$ is similar to that of a uniform base: a base $\mathscr{B}$ is a uniform base if for every point $x$ and every open neighbourhood $U$ of $x$ there are at most finitely many $V \in \mathscr{B}$ for which $x \in V$ and $V \backslash U \neq \varnothing$. It is a result of P. S. Alexandrov [1] that a space is metacompact and developable if and only if it has a uniform base.

The proof of the following lemma is similar to Theorem 4 of [6]

LEMMA 1 . If the space $X$ has $\mathscr{W}$ satisfying uniform open $(\mathrm{G})$ then $X$ is metacompact.

Proof. Suppose that $\kappa$ is a cardinal and that $\left\{U_{\alpha}: \alpha<\kappa\right\}=\mathscr{U}$ is an open cover of $X$. For each $\alpha<\kappa$ let $X_{\alpha}=U_{\alpha} \backslash \bigcup\left\{U_{\beta}: \beta<\alpha\right\}$ and define $O_{\alpha}=\bigcup\left\{V\left(x, U_{\alpha}\right): x \in X_{\alpha}\right\}$. Clearly $O_{\alpha}$ is open and $X_{\alpha} \subseteq O_{\alpha} \subseteq U_{\alpha}$. Hence $\mathcal{O}=\left\{O_{\alpha}: \alpha<\kappa\right\}$ is an open refinement of $\mathscr{U}$ which covers $X$, so it will be sufficient to show that $\mathcal{O}$ is point finite. If $\mathcal{O}$ did not have this property there would exist an $x$ and an increasing sequence $\left\{\alpha_{n}\right\}_{n=0}^{\infty}$ of ordinals such that $x \in O_{\alpha_{n}}$ for each $n$. Since $x \in X_{\alpha}$ for only one $\alpha<\kappa$, we may assume $x \notin X_{\alpha_{i}}$ for any $i$. For each $n$, there is a point $y_{n} \in X_{\alpha_{n}}$ for which $x \in V\left(y_{n}, U_{\alpha_{n}}\right)$. Hence, for each $n$, there exists a $W_{n} \in W(x)$ such that $y_{n} \in W_{n} \subseteq U_{\alpha_{n}}$. For $n>m, y_{n} \notin W_{m}$ since $y_{n} \notin U_{\alpha_{m}}$. Thus $\left\{W_{n}: n \in N\right\}$ is an infinite subset of $W(x)$ and therefore is a local base at $x$. Since $x \notin X_{\alpha_{0}}$, $y_{0} \neq x$ and so $W_{0} \backslash\left\{y_{0}\right\}$ is an open neighbourhood of $x$. Hence there is an $n$ such that $W_{n} \subseteq W_{0} \backslash\left\{y_{0}\right\}$. This leads to a contradiction because $y_{n} \in W_{n}$ for every $n$ and $y_{n} \notin W_{0}$ for every $n>0$.

The following proof of Theorem 1 was suggested to the authors by the referee and simplifies the original proof. It involves the construction of a sieve, similar to the one used to prove that every $\beta$-space (in the sense of Hodel) with a point countable base has a base of countable order [4]. Our notation will be that used by G. Gruenhage in [10].

THEOREM 1. A space $X$ is metacompact and developable if and only if it has $\mathscr{W}$ satisfying uniform open $(\mathrm{G})$

Proof. First suppose that $X$ is metacompact and developable. Let $\left\{\mathscr{H}_{n}\right\}_{n=0}^{\infty}$ be a development. $X$ is metacompact, so we may assume that for each $n, \mathscr{H}_{n+1}$ is a point finite refinement of $\mathscr{H}_{n}$. For each $x \in X$ define

$$
W(x)=\left\{H \in \bigcup\left\{\mathscr{H}_{n}: n \in N\right\}: x \in H\right\} .
$$

It is easily verified that $\mathscr{W}=\{W(x): x \in X\}$ satisfies open (G). To see that it satisfies uniform open (G), consider any $x$ and suppose that $\mathscr{C}$ is an infinite subset of $W(x)$. Let $U$ be any open set containing $x$. There is an $N \in N$ for which $\operatorname{St}\left(x, \mathscr{H}_{N}\right) \subseteq U$ (where $\left.\operatorname{St}\left(x, \mathscr{H}_{N}\right)=\bigcup\left\{H \in \mathscr{H}_{N}: x \in H\right\}\right)$. Because $\left\{H \in \bigcup\left\{\mathscr{H}_{N}: 0 \leqslant n \leqslant N\right\}: x \in H\right\}$ is finite and $\mathscr{C}$ is infinite there must be an $H \in \mathscr{C}$ such that $H \in \mathscr{H}_{n}$ for some $n \geqslant N$, and so $x \in H \subseteq \operatorname{St}\left(x, \mathscr{H}_{N}\right) \subseteq U$. Hence $\mathscr{C}$ is a local base at $x$. This establishes that $\mathscr{W}$ satisfies uniform open $(\mathrm{G})$.

Conversely, suppose that $X$ has $\mathscr{W}$ satisfying uniform open $(\mathrm{G})$. For each $x \in X$, pick an enumeration of $W(x)$ as $\{W(n, x): n \in N\}$ so that if $W(x)$ is infinite there is no repetition and if $W(x)$ is finite (which implies $x$ is isolated) then $W(n, x)=\{x\}$ $=W(m, x)$ whenever $W(n, x)=W(m, x)$ and $n \neq m$. Observe that if $I$ is an infinite subset of $N$ then $\{W(n, x): n \in I\}$ is a local base at $x$.

We shall define a sieve $(G, T)$. Suppose that $\mathscr{T}$ is the topology on $X$ and let $T_{0}=X$. For each $x \in T_{0}$, let $G(x)=W(0, x)$. Inductively define $T_{n} \subseteq X^{n+1}$ and $G: T_{n} \rightarrow \mathscr{T}$ such that for each $n \geqslant 1$

$$
T_{n}=\left\{\left(x_{0}, \ldots, x_{n}\right): t=\left(x_{0}, \ldots, x_{n-1}\right) \in T_{n-1} \text { and } x_{n} \in G(t)\right\},
$$

and

$$
\begin{aligned}
G\left(\left(x_{0}, \ldots, x_{n}\right)\right)=G\left(\left(x_{0}, \ldots, x_{n-1}\right)\right) & \cap V\left(x_{n}, X \backslash\left\{x_{j}: j<n, x_{j} \neq x_{n}\right\}\right) \\
& \cap \bigcap\left\{W\left(i, x_{j}\right): i, j \leqslant n \text { and } x_{n} \in W\left(i, x_{j}\right)\right\} .
\end{aligned}
$$

Let $T=\bigcup_{n=0}^{\infty} T_{n}$ and if $s, t \in T$ define $s \leqslant t$ whenever $s$ is an initial segment of $t .(G, T)$ clearly satisfies the definition of a sieve given in [10]. It will be sufficient to prove that if $b$ is a branch of $T$ and $x \in \bigcap_{t \in b} G(t)$, then $\{G(t): t \in b\}$ is a local base at $x$, since then, by 
[5], $X$ has a base of countable order and thus is developable by [16] because it is metacompact.

So suppose that $b=\left\{t_{n}: n \in N\right\}$ is a branch of $T$ with $t_{n} \in T_{n}$ and $x \in \bigcap_{n=0}^{\infty} G\left(t_{n}\right)$. Then there is a sequence $\left(x_{n}\right)$ such that $t_{n}=\left(x_{0}, \ldots, x_{n}\right)$. We shall first show that $x$ is a cluster point of the sequence $\left(x_{n}\right)$. We can assume that either (1) or (2) below holds:

(1) there is an infinite subset $I$ of $N$ such that $x_{n}=x_{m}$ whenever $n, m \in I$,

(2) there is an infinite subset $J$ of $N$ such that $x_{n} \neq x_{m}$ whenever $n, m \in J$ and $n \neq m$.

If case (1) occurs then if $p=x_{n}$ whenever $n \in I$ then $x=p$ since, whenever $n \in I$,

$$
x \in G\left(t_{n}\right) \subseteq W\left(n, x_{n}\right) \subseteq W(n, p)
$$

and $\{W(n, p): n \in I\}$ is a local base at $p$. If case (2) occurs then for each $n \in J$,

$$
x \in G\left(t_{n}\right) \subseteq V\left(x_{n}, X \backslash\left\{x_{j}: j<n, x_{j} \neq x_{n}\right\}\right) .
$$

Hence there is a $W_{n} \in W(x)$ for which

$$
x_{n} \in W_{n} \subseteq X \backslash\left\{x_{j}: j<n, x_{j} \neq x_{n}\right\} .
$$

Observe that $\left\{W_{n}: n \in J\right\}$ must be an infinite subset of $W(x)$ and thus a local base at $x$. Hence, again by $(\dagger),\left(x_{n}\right)$ clusters at $x$.

We shall now prove that $\left\{G\left(t_{n}\right): n \in N\right\}$ is a local base at $x$. Suppose that $U$ is an open neighbourhood of $x$. Since $V(x, U)$ is an open set containing $x$ there is a $j \in N$ for which $x_{j} \in V(x, U)$. Pick an $i \in N$ such that $x \in W\left(i, x_{j}\right) \subseteq U . W\left(i, x_{j}\right)$ is again an open neighbourhood of $x$ and so there is an $n \geqslant \max \{i, j\}$ such that $x_{n} \in W\left(i, x_{j}\right)$. Hence $x \in G\left(t_{n}\right) \subseteq W\left(i, x_{j}\right) \subseteq U$ and so $\left\{G\left(t_{n}\right): n \in N\right\}$ is a local base for $x$ as required.

2. The point countable base conjecture. Any space with a point countable base has $\mathscr{W}$ satisfying open $(\mathrm{G})$. In this section we investigate under what conditions the converse holds. We first introduce the concept of a pointed open cover. A pointed open cover for a space $X$ with topology $\mathscr{T}$ is a subset $\mathscr{P}$ of $X \times \mathscr{T}$ such that $\{U: \exists x \in X(x, U) \in \mathscr{P}\}$ is a cover for $X . \mathscr{P}$ is said to be point countable if $\{(x, U) \in \mathscr{P}: y \in U\}$ is countable for all $y$, and dense if $y \in \overline{\{x:(x, U) \in \mathscr{P} \wedge y \in U\}}$ for all $y$. The following lemma is central to the discussion.

LEMMA 2. If the space $X$ has $\mathscr{W}$ satisfying open $(G)$ then $X$ has a point countable base if and only if $X$ has a dense, point countable, pointed open cover.

Proof. First suppose that $X$ has a point countable base $\mathscr{B}$. For each non-empty element $U$ of $\mathscr{B}$ pick an $x_{U} \in U$ and define $\mathscr{P}=\left\{\left(x_{U}, U\right): U \in \mathscr{B} \backslash\{\varnothing\}\right\}$. It can be easily verified that $\mathscr{P}$ is a dense, point countable, pointed open cover. Conversely define

$$
\mathscr{B}=\{U \cap W: \exists x(x, U) \in \mathscr{P} \wedge W \in W(x)\} .
$$

Clearly $\mathscr{B}$ is a point countable collection of open sets. To see that $\mathscr{B}$ is a base, consider any $x \in X$ and any open set $O$ containing $x$. Since $\mathscr{P}$ is dense there must exist a $(y, U) \in \mathscr{P}$ such that $x \in U$ and $y \in V(x, O)$. Pick a $W \in W(y)$ for which $x \in W \subseteq O$; then $x \in U \cap W \subseteq O$ and $U \cap W \in \mathscr{B}$. Thus $\mathscr{B}$ is a base as required.
Thus the point countable base conjecture may be restated as

Every space with $\mathscr{W}$ satisfying open $(\mathrm{G})$ has a dense, point countable, pointed open cover.

Hence we now investigate under what conditions a space has a dense, point countable, pointed open cover. Many of the results which follow only require $\mathscr{W}$ to satisfy $(\mathrm{G})$, thus raising the following associated question.

Does every space with $\mathscr{W}$ satisfying $(\mathrm{G})$ have a dense, point countable, pointed open cover?

A small modification to the proof of Lemma 1 yields the next lemma which is required for Theorem 2 .

LemMa 3. If the space $X$ has $\mathscr{W}$ satisfying $(G)$ then $X$ is metaLindelöf.

THEOREM 2. If $X$ is a semi-stratifiable space with $\mathscr{W}$ satisfying $(\mathrm{G})$ then $X$ has a dense, point countable, pointed open cover.

Proof. We shall use the characterisation of semi-stratifiable given by G. D. Creede in [8], which states that a space $X$ with topology $\mathscr{T}$ is semi-stratifiable if and only if there exists a function $g$ from $N \times X$ to $\mathscr{T}$ such that

(1) $\{x\}=\bigcap\{g(n, x): n \in N\}$, and

(2) if $y \in X$ and $\left\{x_{n}\right\}_{n=0}^{\infty}$ is a sequence of points in $X$ such that $y \in g\left(n, x_{n}\right)$ for all $n$, then $\left\{x_{n}\right\}_{n=0}^{\infty}$ converges to $y$.

Let $g$ be such a function. By the previous lemma $X$ is metaLindelöf, so let $\mathscr{U}_{n}$ be a point countable open refinement of $\{g(n, x): x \in X\}$ which covers $X$. For each $U \in \mathscr{U}_{n}$, choose an $x_{U}$ such that $U \subseteq g\left(n, x_{U}\right)$. Define $\mathscr{P}=\left\{\left(x_{U}, U\right): U \in \mathscr{U}_{n} \wedge n \in N\right\}$. Clearly $\mathscr{P}$ is a point countable, pointed open cover. Consider any $y \in X$, for each $n$ pick a $U_{n} \in \mathscr{U}_{n}$ which contains $y$. Letting $x_{n}=x_{U_{n}}$ we see that $y \in g\left(n, x_{n}\right)$. Thus by (2), $\left\{x_{n}\right\}_{n=0}^{\infty}$ converges to $y$. Hence $\mathscr{P}$ is dense since $\left(x_{n}, U_{n}\right) \in \mathscr{P}$ for all $n$.

Notice that the $\mathscr{P}$ produced in the proof of Theorem 2 need not have $x \in U$ when $(x, U) \in \mathscr{P}$. Of course this is not necessary for Lemma 2 to be applicable. However, by modifying the proof of metaLindelöf, see Lemma 4 below, it is possible to obtain this property if desired.

Observe that if the space $X$ has a point countable base $\mathscr{B}$ then, setting $W(x)$ $=\{B \in \mathscr{B}: x \in B\}$ and $\mathscr{W}=\{W(x): x \in X\}, X$ has $\mathscr{W}$ satisfying a stronger condition than open (G), since if $x \in U$ and $U$ is open, then we may take $V(x, U)$ to be an element of $\mathscr{B}$ satisfying $x \in V(x, U) \subseteq U$. Then for any $y \in V(x, U), V(x, U) \in W(y)$ and hence there is a $W \in W(y)$ satisfying $x \in W \subseteq V(x, U)$. By strengthening $(\mathrm{G})$ in this way we can show the existence, for an arbitrary space $X$, of a dense, point countable, pointed open cover.

Formally, if $X$ is a space and $W(x)$ is countable for every $x \in X$ then $\mathscr{W}=\{W(x): x \in X\}$ satisfies $\left(\mathrm{G}^{\prime}\right)$ when it satisfies

$\left(\mathrm{G}^{\prime}\right)$ if $x \in U$ and $U$ is open, then there exists an open $V=V(x, U) \subseteq U$ containing $x$ such that $x \in W \subseteq V$ for some $W \in W(y)$ whenever $y \in V$. 
THEOREM 3. If the space $X$ has $\mathscr{W}$ satisfying $\left(\mathrm{G}^{\prime}\right)$ then $X$ has a dense, point countable, pointed open cover.

Proof. Let $\mathscr{T}$ be the topology on $X$. We shall construct, for each $\alpha \in \omega_{1}$, a subset $\mathscr{P}_{\alpha}$ of $X \times \mathscr{T}$, a subset $X_{\alpha}$ of $X$, and for each $x \in X$ a closed subset $D_{\alpha}^{x}$ of $X$. Suppose that $\mathscr{P}_{\beta}$, $X_{\beta}$ and $D_{\beta}^{x}$ have been constructed for all $\beta<\alpha$ and all $x \in X$. Define

$$
\begin{aligned}
\mathscr{V}_{\alpha} & \left.=\bigcup_{\beta<\alpha} \mathscr{P}_{\beta} \quad \text { (and so } \mathscr{V}_{0}=\varnothing\right), \\
D_{\alpha}^{x} & =\overline{\left\{y: \exists(y, U) \in \mathscr{F}_{\alpha} x \in U\right\}} \quad \text { for each } x, \text { and } \\
X_{\alpha} & =\left\{x: x \in D_{\alpha}^{x}\right\} .
\end{aligned}
$$

Let $\mathscr{P}_{\alpha}$ be a maximal subcollection of $\left\{\left(x, V\left(x, X \backslash D_{\alpha}^{x}\right)\right): x \notin X_{\alpha}\right\}$ such that

$$
\left(x_{1}, V_{1}\right),\left(x_{2}, V_{2}\right) \in \mathscr{P}_{\alpha} \Rightarrow x_{1} \notin V_{2} \vee x_{2} \notin V_{1} .
$$

$\mathscr{P}_{\alpha}$ exists by Zorn's Lemma. Observe that for each $y \in X \backslash X_{\alpha}$ there exists an $(x, V) \in \mathscr{P}_{\alpha}$ such that $y \in V$. Let $\mathscr{P}=\bigcup\left\{\mathscr{P}_{\alpha}: \alpha \in \omega_{1}\right\}$. We shall show that $\mathscr{P}$ is the required dense, point countable, pointed open cover.

First observe that $\mathscr{P}_{0}$ is a pointed open cover. Suppose that $y \in V_{1} \cap V_{2}$ where $\left(x_{i}, V_{i}\right)$ $\in \mathscr{P}_{\alpha}$ for $i=1,2$. We may assume that $x_{1} \notin V_{2}$. By the definition of $\mathscr{P}_{\alpha}$ and since $\mathscr{W}$ satisfies $\left(\mathrm{G}^{\prime}\right)$ there exists, for $i=1,2, W_{i} \in W(y)$ such that $x_{i} \in W_{i} \subseteq V_{i}$. Since $x_{1} \notin V_{2}$, $W_{1} \neq W_{2}$. Thus, since $W(y)$ is countable, $\left\{(x, V) \in \mathscr{P}_{\alpha}: y \in V\right\}$ is countable. Now suppose that $y \in V_{1} \cap V_{2}$ where $\left(x_{1}, V_{1}\right) \in \mathscr{P}_{\alpha},\left(x_{2}, V_{2}\right) \in \mathscr{P}_{\beta}$ and $\alpha<\beta$. As above, for $i=1,2$, there exist $W_{i} \in W(y)$ such that $x_{i} \in W_{i} \subseteq V_{i}$. Suppose that $W_{1}=W_{2}$; then $x_{2} \in V_{1}$ and $x_{1} \in V_{2}$. But $x_{2} \in V_{1}$ implies that $x_{1} \in D_{\beta}^{x_{2}}$, giving $x_{1} \notin V\left(x_{2}, X \backslash D_{\beta}^{x_{2}}\right)=V_{2}$, which is a contradiction. Thus $W_{1} \neq W_{2}$ and hence $\left\{\alpha \in \omega_{1}: \exists(x, V) \in \mathscr{P}_{\alpha} y \in V\right\}$ is countable. Thus $\mathscr{P}$ is point countable. Furthermore, $X=\bigcup\left\{X_{\alpha}: \alpha \in \omega_{1}\right\}$ since if $y \notin X_{\alpha}$ then there exists an $(x, V) \in \mathscr{P}_{\alpha}$ such that $y \in V$. But $X=\bigcup\left\{X_{\alpha}: \alpha \in \omega_{1}\right\}$ implies that $x \in \overline{\{y: \exists(y, V) \in \mathscr{P} x \in V\}}$ for all $x$. Hence $\mathscr{P}$ is dense.

In [7] it was shown that any separable space with $\mathscr{W}$ satisfying open (G) has a countable base. The following theorem establishes that any space with density $\leqslant \omega_{1}$ and with $\mathscr{W}$ satisfying open $(G)$ has a point countable base.

THEOREM 4. If the first countable space $X$ has density $\leqslant \omega_{1}$ then $X$ has a dense, point countable, pointed open cover.

Proof. Suppose that $\left\{x_{\alpha}: \alpha<\omega_{1}\right\}$ is a dense subset of $X$. Let $C_{\alpha}=\overline{\left\{x_{\beta}: \beta<\alpha\right\}}$ for each $\alpha<\omega_{1}$ and let

$$
\mathscr{P}=\left\{\left(x_{\alpha}, X \backslash C_{\alpha}\right): \alpha<\omega_{1}\right\}
$$

Observe that $C_{0}=\varnothing$ and so $\mathscr{P}$ is a pointed open cover. We shall show that $\mathscr{P}$ is dense and point countable. Consider any $x \in X$, let $\{R(n, x): n \in N\}$ be a local base at $X$. For each $n$ there is an $\alpha_{n}<\omega_{1}$ such that $x_{\alpha_{n}} \in R(n, x)$. Clearly $x \in C_{\alpha}$ where $\alpha=\sup \left\{\alpha_{n}\right.$ : $n \in N\}<\omega_{1}$. Thus $\mathscr{P}$ is point countable since $x \notin X \backslash C_{\beta}$ for any $\beta \geqslant \alpha$. Furthermore, $\mathscr{P}$ is clearly dense, since if $\alpha$ is the least ordinal such that $x \in C_{\alpha}$ then $x \in \overline{\left\{x_{\beta}: x \in X \backslash C_{\beta}, \beta<\alpha\right\}}$. Thus $\mathscr{P}$ is dense.

The above theorem gives us some insight into the structure of any possible counterexample to the conjecture. The following two theorems give us further insight. The first shows that any space with $\mathscr{W}$ satisfying open $(G)$ has a dense subset which has a point countable base.

LEMMA 4. Suppose the space $X$, with topology $\mathscr{T}$, has $\mathscr{W}$ satisfying $(\mathrm{G})$. Further suppose that $Y \subseteq X$ and that for each $y \in Y$ there is an open set (in $X) U_{y}$ which contains $y$. Then there exists a subset $\mathscr{P}$ of $Y \times \mathscr{T}$ which satisfies

(1) $\{(y, U) \in \mathscr{P}: x \in U\}$ is countable for all $x \in X$,

(2) $Y \subseteq \bigcup\{U: \exists y \in Y(y, U) \in \mathscr{P}\}$, and

(३) $(y, U) \in \mathscr{P} \Rightarrow y \in U \subseteq U_{y}$.

Proof. Suppose that $\kappa$ is a cardinal and $Y=\left\{y_{\alpha}: \alpha<\kappa\right\}$. We shall construct for each $\alpha<\kappa$ an open set $O_{\alpha}$ of $X$. Suppose that $O_{\beta}$ has been constructed for each $\beta<\alpha$. If $y_{\alpha} \in \bigcup\left\{O_{\beta}: \beta<\alpha\right\}$ then define $O_{\alpha}=\varnothing$, otherwise define

$$
O_{\alpha}=\bigcup\left\{V\left(x, U_{y_{\alpha}}\right): x \in U_{y_{\alpha}} \backslash \bigcup_{\beta<\alpha} O_{\beta}\right\} .
$$

Define $\mathscr{P}=\left\{\left(y_{\alpha}, O_{\alpha}\right): O_{\alpha} \neq \varnothing\right\}$. Clearly $\mathscr{P}$ satisfies conditions (2) and (3). Suppose that $x \in O_{\alpha}, O_{\beta}$ where $\alpha<\beta$. Then there exists an $x_{\alpha} \in U_{y_{\alpha}} \backslash \bigcup_{\gamma<\alpha} O_{\gamma}$ and an $x_{\beta} \in U_{y_{\beta}} \backslash \bigcup_{\gamma<\beta} O_{\gamma}$ such that $x \in V\left(x_{\alpha}, U_{y_{\alpha}}\right)$ and $x \in V\left(x_{\beta}, U_{y_{\beta}}\right)$. Pick $W_{1}, W_{2} \in W(x)$ satisfying $x_{\alpha} \in W_{1} \subseteq U_{y_{\alpha}}$ and $x_{\beta} \in W_{2} \subseteq U_{y_{\beta}}$. If $W_{1}=W_{2}$ then $x_{\beta} \in U_{y_{\alpha}} \backslash \bigcup_{\gamma<\alpha} O_{\gamma} \subseteq O_{\alpha}$, which is a contradiction. Thus $\left\{\alpha: x \in O_{\alpha}\right\}$ is countable since $W(x)$ is.

THEOREM 5. If the space $X$ has $\mathscr{W}$ satisfying $(G)$ then it has a point countable, pointed open cover $\mathscr{P}$ such that $x \in U$ whenever $(x, U) \in \mathscr{P}$ and $\{x \in X: \exists U(x, U) \in \mathscr{P}\}$ is dense in $X$.

Proof. For each $\alpha<\omega_{1}$ we shall construct a subset $\mathscr{P}_{\alpha}$ of $X \times \mathscr{T}$ (where $\mathscr{T}$ is the topology on $X$ ) and a subset $X_{\alpha}$ of $X$. Suppose that $\mathscr{P}_{\beta}$ and $X_{\beta}$ have been defined for all $\beta<\alpha$. Set

$$
X_{\alpha}=\left\{x ; \exists U(x, U) \in \mathscr{P}_{\beta}, \beta<\alpha\right\},
$$

and so in particular $X_{0}=\varnothing$. By the previous lemma, putting $Y=X \backslash \overline{X_{\alpha}}$ and $U_{y}=V(y, Y)$ for each $y \in Y$, there exists a subset $\mathscr{P}_{\alpha}$ of $\left(X \backslash \overline{X_{\alpha}}\right) \times \mathscr{T}$ which satisfies

(1) $\left\{(y, U) \in \mathscr{P}_{\alpha}: x \in U\right\}$ is countable for all $x \in X$,

(2) $X \backslash \overline{X_{\alpha}} \subseteq \bigcup\left\{U: \exists y \in X \backslash \overline{X_{\alpha}}(y, U) \in \mathscr{P}_{\alpha}\right\}$, and

(3) $(y, U) \in \mathscr{P}_{\alpha} \Rightarrow y \in U \subseteq U_{y}=V\left(y, X \backslash \overline{X_{\alpha}}\right)$.

Define $\mathscr{P}=\bigcup\left\{\mathscr{P}_{\alpha}: \alpha<\omega_{1}\right\}$. We shall show that $\mathscr{P}$ is a point countable, pointed open cover. First observe that $\mathscr{P}_{0}$ is a pointed open cover. Now, suppose that $y \in X$ and there exist $\left(x_{1}, U_{1}\right) \in \mathscr{P}_{\alpha}$ and $\left(x_{2}, U_{2}\right) \in \mathscr{P}_{\beta}$ such that $y \in U_{1} \cap U_{2}$ and $\alpha<\beta$. Pick $W_{1}, W_{2}$ $\in W(y)$ satisfying $x_{1} \in W_{1} \subseteq X \backslash \overline{X_{\alpha}}$ and $x_{2} \in W_{2} \subseteq X \backslash \overline{X_{\beta}}$. Since $x_{1} \in X_{\beta}, W_{1} \neq W_{2}$, and 
therefore $\left\{\alpha: \exists(x, U) \in \mathscr{P}_{\alpha} y \in U\right\}$ is countable. This combined with (1) above implies that $\mathscr{P}$ is point countable. Notice that by $(3), x \in U$ whenever $(x, U) \in \mathscr{P}$. Finally observe that if $y \notin \overline{X_{\alpha}}$ then there is an $(x, U) \in \mathscr{P}_{\alpha}$ such that $y \in U$. Therefore $X=\bigcup\left\{\overline{X_{\alpha}}: \alpha \in \omega_{1}\right\}$ and so $X=\overline{\{x: \exists U(x, U) \in \mathscr{P}\}}$.

Taking $Y=\{x \in X: \exists U(x, U) \in \mathscr{P}\}$ in the above theorem we obtain, by Lemma 2, the following corollary.

COROLlary. If the space $X$ has $\mathscr{W}$ satisfying open $(\mathrm{G})$ then it has a dense subset $Y$ which has a point countable base.

Our next theorem establishes that a counterexample cannot be constructed by taking a locally countable union of spaces each of which has a point countable base.

THEOREM 6. Suppose that the space $X$ has $\mathscr{W}$ satisfying $(\mathrm{G})$ and is the locally countable union of subspaces $Y_{i}(i \in I)$, each of which has a dense, point countable, pointed open cover. Then $X$ also has a dense, point countable, point open cover.

Proof. For each $x \in X$, there is an open neighbourhood $N_{x}$ of $x$ which meets only countably many of the $Y_{i}$. Let $\mathscr{P}_{i}$ be a dense, point countable, pointed open cover of $Y_{i}$. Fix an $i \in I$, for each $(y, U) \in \mathscr{P}_{i}$ pick a $U^{\prime}$, open in $X$, such that $U^{\prime} \cap Y_{i}=U$ and define

$$
S_{i}(y, U)=\bigcup\left\{V\left(x, U^{\prime} \cap N_{x}\right): x \in U\right\} .
$$

Observe that $S_{i}(y, U)$ is open in $X$ and that $S_{i}(y, U) \cap Y_{i}=U$. Define

$$
\mathscr{P}_{i}^{\prime}=\left\{\left(y, S_{i}(y, U)\right):(y, U) \in \mathscr{P}_{i}\right\}
$$

and set $\mathscr{P}=\left\{\mathscr{P}_{i}^{\prime}: i \in I\right\}$. We shall show that $\mathscr{P}$ is the required dense, point countable pointed open cover. Clearly, since $X=\bigcup\left\{Y_{i}: i \in I\right\}, \mathscr{P}$ is a pointed open cover. In order to show that $\mathscr{P}$ is point countable we first show that $\left\{(y, O) \in \mathscr{P}_{i}^{\prime}: x \in O\right\}$ is countable for each $x \in X$ and $i \in I$. Observe that if $\left\{(y, O) \in \mathscr{P}_{i}^{\prime}: x \in O\right\}$ is uncountable then there must exist an uncountable subset $A$ of $\mathscr{P}_{i}$ such that $x \in S_{i}(y, U)$ for every $(y, U) \in A$. For each $(y, U) \in A$ pick a $z_{(y, U)} \in U$ such that $x \in V\left(z_{(y, U)}, U^{\prime} \cap N_{\left.z_{(y, U}\right)}\right)$. There is a $W_{(y, U)} \in W(x)$ which satisfies

$$
z_{(y, U)} \in W_{(y, U)} \subseteq U^{\prime} \cap N_{z(y, U)} \subseteq U^{\prime} .
$$

Since $W(x)$ is countable we may assume that $W_{\left(y_{1}, U_{1}\right)}=W_{\left(y_{2}, U_{2}\right)}$ whenever $\left(y_{j}, U_{j}\right) \in A$ for $j=1,2$. Thus fixing a $\left(y_{1}, U_{1}\right) \in A$ we see that $z_{\left(y_{1}, U_{1}\right)} \in U^{\prime} \cap Y_{i}=U$ for every $(y, U) \in A$, which contradicts $\mathscr{P}_{i}$ being point countable. Hence $\left\{(y, O) \in \mathscr{P}_{i}^{\prime}: x \in O\right\}$ is countable as required.

So suppose $\mathscr{P}$ is not point countable. Then from the above there is an $x \in X$ and an uncountable subset $I_{1}$ of $I$ such that for each $i \in I_{1}$ there is an element $\left(y_{i}, U_{i}\right)$ in $\mathscr{P}_{i}$ such that $x \in S_{i}\left(y_{i}, U_{i}\right)$. Hence there is a $z_{i} \in U_{i}$ such that $x \in V\left(z_{i}, U_{i}^{\prime} \cap N_{z_{i}}\right)$. Since $W(x)$ is countable there is an uncountable subset $I_{2}$ of $I_{1}$ such that for all $i, j \in I_{2}$, $z_{i} \in U_{j}^{\prime} \cap N_{z_{j}} \subseteq N_{z_{j}}$. But then $N_{z_{i}} \cap Y_{j} \neq \varnothing$ for all $i, j \in I_{2}$ and this contradicts the definition of $N_{z i}$. Therefore $\mathscr{P}$ is point countable as required. Finally, we must show that
$\mathscr{P}$ is dense; this follows easily from the fact that each $\mathscr{P}_{i}$ is dense (in $Y_{i}$ ) and each $x$ in $X$ lies in $Y_{i}$ for some $i$.

We now bring together the results of this section stating them in terms of point countable bases.

THEOREM 7. Suppose the space $X$ has $\mathscr{W}$ satisfying open $(\mathrm{G})$. Then if $X$ also satisfies one of the following then $X$ has a point countable base:

(1) $X$ is semi-stratifiable,

(2) $X$ has $\mathscr{W}^{\prime}$ satisfying $\left(\mathrm{G}^{\prime}\right)\left({ }^{2}\right)$,

(3) $X$ has density $\leqslant \omega_{1}$,

(4) $X$ is the locally countable union of subspaces each of which has a point countable hase.

The referee observed that (1) in the above may be strengthened to $\beta$-spaces. A modification of the proof of Theorem 1 yields that any $\beta$-space with $\mathscr{W}$ satisfying open $(\mathrm{G})$ has a base of countable order (cf. 2.10 of [4]). Observe that a space with $\mathscr{W}$ satisfying open $(\mathrm{G})$ is hereditarily metaLindelöf and thus it can be seen that any $\beta$-space with $\mathscr{W}$ satisfying open $(\mathrm{G})$ has a point countable base.

In addition to the above theorem P. J. Nyikos and A. W. Roscoe have established the following result.

THEOREM 8. If $X$ is a first countable, non-archimedean space which has $\mathscr{W}$ satisfying (G), then $X$ has a point countable base.

Our final theorem of this section demonstrates how unpleasant any counterexample must be and yields a partial result for scattered spaces.

THEOREM 9. If the space $X$ is a counterexample to the conjecture then there is a non-empty subspace $X^{\prime}$ of $X$, every non-empty open subset of which is a counterexample.

Proof. Let $\mathscr{U}=\{U \subseteq X: U$ is open in $X$ and has a point countable base $\}$ and define $Y=\bigcup \mathscr{U}$. By Lemma 3, $Y$ is metaLindelöf and therefore $Y$ can be expressed as the union of a point countable collection of open sets each of which has a point countable base and so $Y$ has a point countable base. Hence by Theorem $7, X^{\prime}=X \backslash Y$ does not have a point countable base. Suppose that $U$ is a subset of $X^{\prime}$ which is open in $X^{\prime}$ and has a point countable base. Since $X^{\prime}$ is closed in $X, X^{\prime} \backslash U$ is closed in $X$ and so $Y \cup U=X \backslash\left(X^{\prime} \backslash U\right)$ is open in $X$. But by Theorem 7, $Y \cup U$ has a point countable base and so $Y \cup U \in \mathscr{\%}$, which implies that $U \subseteq Y$ and hence $U=\varnothing$.

Recall that a space $X$ is scattered if every non-empty subspace has an isolated point (in the subspace topology). Hence, in particular, every non-empty subspace of $X$ has an -open subset which has a point countable base. Therefore by Theorem 9 we have the following result.

THEOREM 10. If $X$ is a scattered space with $\mathscr{W}$ satisfying open $(G)$ then $X$ has a point countable base.

$\left({ }^{2}\right)$ Of course $\mathscr{W}^{\prime}$ may be equal to $\mathscr{W}$. 
3. When $\mathscr{W}$ satisfies chain (F). In [6] it was established that a space with $\mathscr{W}$ satisfying chain $(\mathrm{F})$ is monotonically normal. The converse is known to be true for stratifiable spaces (see [6], p. 493), it being an open question as to whether the converse is true in general. In the first part of this section we shall investigate this question.

We begin by introducing the concept of an acyclic monotonically normal operator. An acyclic monotonically normal operator is an operator which assigns to each $x$ and open set $U$ containing $x$ an open set $V(x, U)$ containing $x$ which satisfies

(1) $V(x, U) \subseteq V\left(x, U^{\prime}\right)$ whenever $U \subseteq U^{\prime}$,

(2) $V(x, X \backslash\{y\}) \cap V(y, X \backslash\{x\})=\varnothing$ if $x \neq y$, and

(3) $\bigcap_{i=0}^{n-1} V\left(x_{i}, X \backslash\left\{x_{i+1}\right\}\right)=\varnothing$ whenever $n \geqslant 2, x_{0}, \ldots, x_{n-1}$ are distinct and $x_{n}=x_{0}$.

Observe that (3) implies (2) and that (1) and (2) are precisely the conditions for a space to be monotonically normal. Our next theorem establishes that the existence of an acyclic monotonically normal operator is equivalent to the existence of $\mathscr{W}$ satisfying chain (F). Hence the question of whether any monotonically normal space has $\mathscr{W}$ satisfying chain $(\mathbf{F})$ may be rewritten as

Does every monotonically normal space have an acyclic monotonically normal operator?

We note that there is a monotonically normal operator on the four point discrete space, which is not acyclic. Of course any discrete space has an acyclic monotonically normal operator.

THEOREM 11. A space $X$ has an acyclic monotonically normal operator if and only if $X$ has $\mathscr{W}$ satisfying chain $(\mathrm{F})$.

Proof. It can be easily verified that the operator defined in [6] when $X$ has $\mathscr{W}$ satisfying chain $(F)$ is an acyclic monotonically normal operator.

Conversely, fix a point $a \in X$. We shall define a "nearness" relation on $X$ as follows: For $x \neq y$,

$$
x \sim_{a} y \Leftrightarrow a \in V(x, X \backslash\{y\}) \quad \text { (" } x \text { is nearer to } a \text { than } y \text { is"). }
$$

Observe that $x \sim_{a} y$ implies that $y \sim_{a} x$, that $x \sim_{a} x$ for every $x \in X$ and that $a \sim_{a} x$ for every $x \neq a$. Define the relation $<_{a}$ by

$$
x<{ }_{a} y \Leftrightarrow\left(\begin{array}{l}
\exists n \geqslant 1, x_{0}, \ldots, x_{n} \text { such that } x=x_{0}, y=x_{n} \\
\text { and } x_{i} \sim_{a} x_{i+1} \text { for } i=0, \ldots, n-1
\end{array}\right) .
$$

$<_{a}$ is the transitive closure of $\sim_{a}$ and, by (3), $<_{a}$ is an irreflexive partial order. By Zorn's Lemma there is an irreflexive linear order $\triangleleft_{a}$ on $X$ satisfying

$$
x<_{a} y \Rightarrow x \triangleleft_{a} y .
$$

Defining $W(a)=\left\{S_{a}(x): x \in X\right\}$, where $S_{a}(x)=\left\{y: y \triangleleft_{a} x\right\} \cup\{x\}$, we see that $W(a)$ is a collection of sets, each containing $a$, which is linearly ordered with respect to inclusion. It only remains to show that $\mathscr{W}=\{W(x): x \in X\}$ satisfies $(\mathrm{F})$. Suppose $x$ is an element of the open set $U$; then $x \in V(x, U) \subseteq U$ (where $V(\cdot, \cdot)$ is the acyclic monotonically normal operator). Consider any $y \in V(x, U)$. The proof will be complete if we can show that $x \in S_{y}(x) \subseteq U$. By definition, $x \in S_{y}(x)$ and if $z \notin U$ then $V(x, U) \subseteq V(x, X \backslash\{z\})$, thus $y \in V(x, X \backslash\{z\})$. Hence $x \triangleleft_{y} z$ and since $\triangleleft_{y}$ is a linear order, $z \notin S_{y}(x)$, thus $S_{y}(x) \subseteq U$.

We claim that the monotonically normal operator defined by R. W. Heath, D. J. Lutzer and P. L. Zenor on a linearly ordered space [11] is an acyclic monotonically normal operator. In addition it can be shown that H. Tamano and J. E. Vaughan's elastic spaces [14], and hence Vaughan's linearly stratifiable spaces [15], have an acyclic monotonically normal operator (C. R. Borges has shown that such spaces are monotonically normal [3]). We can therefore deduce the following result.

THEOREM 12. If $X$ is either a generalised order space or an elastic space then $X$ has $\mathscr{W}$ satisfying chain (F).

A space $X$ is said to be a $\mathrm{K}_{0}$-space if for every subspace $Y$ of $X$ there is a function $\kappa$ from $\mathscr{T}_{Y}$ (the subspace topology on $Y$ ) to $\mathscr{T}_{X}$ (the topology on $X$ ) satisfying:

(1) $Y \cap \kappa(U)=U$ for each $U \in \mathscr{T}_{Y}$,

(2) $\kappa(U) \cap \kappa(V)=\kappa(U \cap V)$ for each $U, V \in \mathscr{T}_{Y}$, and

(3) $\kappa(\varnothing)=\varnothing$.

The concept of a $\mathrm{K}_{0}$-space is due to K. Kuratowski [12, $\left.\S 21, \mathrm{XI}, \mathrm{p} .226\right]$ where he shows that every metrisable space is a $\mathrm{K}_{0}$-space. In addition to metrisable spaces, it is known that generalised order spaces and retractifiable spaces are $\mathrm{K}_{0}$-spaces. It is a question of E. K. van Douwen [9] as to whether every monotonically normal space is a $\mathrm{K}_{0}$-space. We have the following result.

THEOREM 13. If the space $X$ has $\mathscr{W}$ satisfying chain $(\mathrm{F})$ then $X$ is a $K_{0}$-space.

Proof. For each open subset $U$ of $X$ and each subset $A$ of $U$ define

$$
H(A, U)=\{y \in X: \exists W \in W(y)(W \subseteq U) \wedge(W \cap A \neq \varnothing)\} .
$$

Observe that $A \subseteq H(A, U)^{\circ}$ since $\bigcup\{V(x, U): x \in A\} \subseteq H(A, U)^{\circ}$. Let $Y$ be any subspace of $X$. Define $\kappa$ from $\mathscr{T}_{Y}$ to $\mathscr{T}_{X}$ by $\kappa(U)=H\left(U, X \backslash(\overline{Y \backslash U)})^{\circ} . \kappa\right.$ is well defined since $U \subseteq X \backslash(\overline{Y \backslash U})$ for each $U \in \mathscr{T}_{Y}$. We claim that $\kappa$ satisfies (1)-(3) of the above definition. The only difficulty is in establishing $\kappa(U) \cap \kappa(V) \subseteq \kappa(U \cap V)$. So consider any $y \in \kappa(U) \cap \kappa(V)$. Since $y \in \kappa(U)$, there exists a $W_{1} \in W(y)$ such that $W_{1} \subseteq X \backslash \overline{(Y \backslash U)}$ and $W_{1} \cap U \neq \varnothing$. Similarly, there is a $W_{2} \in W(y)$ such that $W_{2} \subseteq W \backslash(\overline{Y \backslash V})$ and $W_{2} \cap V \neq \varnothing$. $W(y)$ is linearly ordered with respect to inclusion, so we may assume, without loss of generality, that $W_{1} \subseteq W_{2}$. Notice that

$$
W_{1} \cap U \subseteq W_{2} \cap U \subseteq(X \backslash(\overline{Y \backslash V})) \cap Y=V .
$$

So in particular $W_{1} \cap(U \cap V) \neq \varnothing$. Observe that

$$
(X \backslash(\overline{Y \backslash U})) \cap(X \backslash(\overline{Y \backslash V})) \subseteq X \backslash(\overline{Y \backslash(U \cap V)}),
$$


thus $W_{1} \subseteq X \backslash(\overline{Y \backslash(U \cap V)})$ and so $y \in H(U \cap V, X \backslash(\overline{Y \backslash(U \cap V))})$. But $\kappa(U) \cap \kappa(V)$ is open and so $\kappa(U) \cap \kappa(V) \subseteq \kappa(U \cap V)$.

The above result yields the following interesting question.

Does every monotonically normal $\mathrm{K}_{0}$-space have an acyclic monotonically normal operator?

We finish by stating the "unified paracompactness theorem" which was indicated in [6]. In [6] it was established that

(1) If the space $X$ has $\mathscr{W}$ satisfying chain (F) and each $W(x)$ is well ordered by $\supseteq$, then $X$ is paracompact.

(2) If the space $X$ has $\mathscr{W}$ satisfying chain (F) and each $W(x)$ consists of neighbourhoods of $x$, then $X$ is paracompact.

Clearly both these results may be deduced from the following theorem.

THEOREM 14. If the space $X$ has $\mathscr{W}$ satisfying chain $(\mathrm{F})$ and for each $x, W(x)$ $=W_{1}(x) \cup W_{2}(x)$ where $W_{1}(x)$ consists of neighbourhoods of $x$ and $W_{2}(x)$ is well ordered by $\supseteq$, then $X$ is paracompact.

The Sorgenfrey line [13] is an example of a space which satisfies the hypotheses of Theorem 14, but neither (1) nor (2) above.

\section{References}

[1] P. S. Alexandrov, On the metrisation of topological spaces, Bull. Acad. Polon. Sci. 8 (1960), $135-140$ (in Russian).

[2] R. H. Bing, Metrization of topological spaces, Canad. J. Math. 3 (1951), 175-186

[3] C. R. Borges, A study of monotonically normal spaces, Proc. Amer. Math. Soc. 38 (1973) 211-214.

[4] J. Chaber, On point-countable collections and monotonic properties, Fund. Math. 94 (1977), 209-219.

[5] J. Chaber, M. M. Coban and K. Nagami, On monotonic generalizations of Moore spaces, Cech complete spaces and p-spaces, Fund. Math. 84 (1974), 107-119.

[6] P. J. Collins, G. M. Reed, A. W. Roscoe and M. E. Rudin, A lattice of conditions on topological spaces, Proc. Amer. Math. Soc. 94 (1985), 487-496.

[7] P. J. Collins and A. W. Roscoe, Criteria for metrisability, Proc. Amer. Math. Soc. 90 (1984) $631-640$

[8] G. D. Creede, Concerning semi-stratifiable spaces, Pacific J. Math. 32 (1970), 47-54.

[9] E. K. van Douwen, Simultaneous extension of continuous functions, Thesis, Vrije Universiteit, Amsterdam 1975.

10] G. Gruenhage, Generalized metric spaces, in: Handbook of Set-Theoretic Topology, K. Kunen and J. E. Vaughan (eds.), North-Holland, 1984, 423-501.

[11] R. W. Heath, D. J. Lutzer and P. L. Zenor, Monotonically normal spaces, Trans. Amer. Math. Soc. 178 (1973), 481-493.

[12] K. Kuratowski, Topology, Vol. I, Academic Press, New York 1966.
[13] R. H. Sorgenfrey, On the topological product of paracompact spaces, Bull. Amer. Math. Soc. 53 (1947), 631-632.

[14] H. T amano and J. E. V a ughan, Paracompactness and elastic spaces, Proc. Amer. Math. Soc. 28 (1971), 299-303.

[15] J. E. Vaughan, Linearly stratifiable spaces, Pacific J. Math. 43 (1972), 253-266.

[16] J. M. Worrell, Jr. and H. H. Wicke, Characterizations of developable topological spaces, Canad. J. Math. 17 (1965), 820-830.

\section{P. J. Moody}

TRINITY COLLEGE

Oxford OX1 $3 \mathrm{BH}$

England

DEPARTMENT OF MATHEMATICS

Athen, Ohi $45701-2979$

G. M. Reed and P. J. Collins

\section{ST. EDMUND HALL}

Oxford OX1 4BH

England
A. W. Roscoe

UNIVERSITY COLLEGE Oxford OX1 4AR 